\title{
Single-axis combined shearography and digital speckle photography instrument for full surface strain characterization
}

\author{
Roger M. Groves, MEMBER SPIE \\ Cranfield University \\ Optical Sensors Group \\ Centre for Photonics and Optical \\ Engineering \\ School of Engineering \\ Cranfield, Bedfordshire, MK43 OAL \\ United Kingdom \\ Shan Fu \\ Cranfield University \\ Centre for Computer Vision and Image \\ Processing \\ School of Engineering \\ Cranfield, Bedfordshire, MK43 OAL \\ United Kingdom \\ Stephen W. James \\ Ralph P. Tatam, MEMBER SPIE \\ Cranfield University \\ Optical Sensors Group \\ Centre for Photonics and Optical \\ Engineering \\ School of Engineering \\ Cranfield, Bedfordshire, MK43 OAL \\ United Kingdom \\ E-mail: r.p.tatam@cranfield.ac.uk
}

\begin{abstract}
Full characterization of the surface strain requires the measurement of six displacement gradient components of the surface strain tensor. The out-of-plane displacement gradient component may be directly measured using the full-field speckle interferometry technique of shearography, but to fully characterize the surface strain using shearography, a minimum of three illumination, or viewing, directions are required. The image processing technique of digital speckle photography (DSP) is sensitive to in-plane displacement for normal collinear illumination and viewing, with the displacement gradient components obtained by differentiation. A combination of shearography and digital speckle photography is used to perform full characterization of the surface strain using a single illumination and viewing direction. The increase in complexity compared with a standard single-channel shearography system lies predominantly in the additional image processing requirements. Digital speckle photography image processing is performed using the optical flow field technique and the advantages of this technique compared with correlation are discussed. The design of the instrument is described and full surface strain measurements made with the system are presented. (c) 2005 Society of Photo-Optical Instrumentation Engineers. [DOI: 10.1117/1.1842779]
\end{abstract}

Subject terms: strain measurement; limited optical access; shearography; digital speckle photography.

Paper 040207 received Apr. 15, 2004; revised manuscript received Jul. 7, 2004; accepted for publication Jul. 7, 2004; published online Jan. 13, 2005.

\section{Introduction}

Shearography, ${ }^{1,2}$ also known as speckle shearing interferometry, is a full-field noncontact optical technique, sensitive to the gradient of displacement. Shearography is usually used for qualitative investigation of surface and subsurface defects. A speckle pattern is formed by illuminating an optically rough surface by an expanded laser beam. This speckle pattern is optically mixed with an identical, but displaced, speckle pattern using a shearing device, such as a shearing Michelson interferometer, ${ }^{1}$ forming a speckle interferogram, that can be recorded by a camera. Correlation of speckle interferograms recorded before and after object deformation yields correlation fringes sensitive to the gradient of displacement.

Digital speckle photography (DSP) ${ }^{3-6}$ can be used to determine the displacement of speckles in the plane of the camera image. As in the case of shearography, a speckle pattern is formed by illuminating an optically rough surface by an expanded laser beam. This speckle pattern is not optically mixed, but is recorded directly by the camera. A second speckle pattern is recorded after object deformation. The displacement fields may be determined using either digital speckle photography correlation techniques (SPC) ${ }^{6}$ or optical flow field techniques (OFF). ${ }^{7}$ The displacement field is subsequently differentiated to yield the displace-

0091-3286/2005/\$22.00 @ 2005 SPIE ment gradient. Previously, DSP has been used to measure the magnitude of the applied shear in shearography ${ }^{8}$ and to compensate for bulk object motion before correlation of interferograms in speckle interferometry. ${ }^{9}$

Full characterization of the surface strain requires the measurement of six components of displacement gradient. A full surface strain measurement may be made using shearography. ${ }^{10}$ This requires illumination, or viewing, of the surface under investigation from three or more directions, ${ }^{11}$ and the use of two directions of applied shear. Each measurement channel, in general, is sensitive to a mixture of the in-plane and out-of-plane displacement gradient components. The in-plane and out-of-plane displacement gradient components are obtained by a coordinate transformation. However, to minimize errors in the coordinate transformation process, angles of illumination of 20 deg or more from the normal to the object surface would have to be employed. ${ }^{12}$ For this reason, a shearography instrument employing single-axis illumination and viewing would only be able to measure the out-of-plane displacement gradient components. Typically, shearography has a sensitivity of the order of tens of microstrain. DSP can determine the in-plane displacement components directly, but the determination of the out-of-plane component requires multiple viewing directions using a stereoscopic technique. ${ }^{13}$ For illumination and viewing normal to the object surface, only the in-plane displacement, and, by differentiation, the in-plane displacement gradient compo- 
nents, can be measured using DSP. The DSP technique has a lower sensitivity than shearography, and is of the order of hundreds of microstrain.

In this work, shearography is used to measure the outof-plane displacement gradient components, while the DSP image processing technique of optical flow field is used to measure the in-plane displacement gradient components. Experimental measurements of full surface strain measurement are performed using the combined shearography and digital speckle photography instrument.

\section{Shearography}

The full-field optical technique of shearography is sensitive to a displacement gradient component, which is determined by the sensitivity vector, which is the bisector of the illumination and viewing direction vectors, and the direction of applied shear. The sensitivity is adjustable by changing the magnitude of shear applied using the shearing element in the interferometer.

The out-of-plane displacement gradient can be measured directly by using collinear illumination and viewing normal to the object surface. ${ }^{14}$ Illumination at a small angle to the normal is usually employed, as this is easier to implement experimentally. This configuration is still highly sensitive to the out-of-plane displacement gradient component, and is employed in the experimental section of this work.

For this configuration, the sensitivity to the out-of-plane displacement gradient $\delta w / \delta x$ is given by:

$\Delta \phi=\frac{4 \pi}{\lambda \cos \theta} \frac{\delta w}{\delta x} d x$

where $\Delta \phi$ is the optical phase change, $\lambda$ is the optical wavelength, $d x$ is the magnitude of applied shear, $\theta$ is the angle between the illumination direction and the normal to the object surface, and $\cos \theta \sim 1.0$. A similar expression may be obtained for a shear applied in the orthogonal $y$ direction. The magnitude of the applied shear lies typically between 1 and $10 \mathrm{~mm}$.

Phase-stepping techniques ${ }^{15}$ are commonly used to extract the phase information from interferograms in speckle interferometry. This technique eliminates ambiguity in the direction of phase change due to the object deformation. In this work, a three-step $2 \pi / 3$ radians phase-step algorithm is used. The phase $\Delta \phi$ is calculated using:

$\Delta \phi=\sqrt{3} \frac{\left(\left|D-R_{1}\right|-\left|D-R_{3}\right|\right)}{\left(2\left|D-R_{2}\right|-\left|D-R_{1}\right|-\left|D-R_{3}\right|\right)}$,

where $R_{1}, R_{2}$, and $R_{3}$ are phase-stepped reference images for the phase steps $-2 \pi / 3,0$, and $2 \pi / 3$ radians, respectively, recorded before object deformation, and $D$ is the image recorded at the phase step of 0 radians after object deformation.

\section{Digital Speckle Photography}

The experimental considerations are identical for both the DSP techniques of SPC $^{6}$ and OFF. ${ }^{7}$ The optically rough surface of the object is illuminated by an expanded laser beam forming a speckle pattern. Speckle patterns recorded by a camera, before and after object deformation, are pro- cessed to yield the in-plane displacement. The difference between the SPC and OFF techniques lies in the approach to the image processing.

Speckle pattern correlation is an established technique for the investigation of speckle patterns. However, previous work $^{16-18}$ has identified issues regarding the selection of the size of the correlation window and the necessity to interpolate to achieve subpixel accuracy in the analysis. The technique is computationally intensive.

In this work, the OFF technique is used to determine the in-plane displacement. The OFF technique is defined as the displacement distribution of the apparent motion of the intensity pattern on the image plane of the observer. ${ }^{7}$ It is assumed that the change in pixel intensity values is solely due to the motion of the speckle pattern, and that the motion of the speckle pattern is directly related to the displacement of the surface, as stated in Eq. (3):

$E(x, y, t)=E(x+u, y+v, t+\Delta t)$,

where $E(x, y, t)$ is the intensity distribution at time $t$ and $E(x+u, y+v, t+\Delta t)$ is the intensity distribution at time $t$ $+\Delta t$. The intensity of the point $(x, y)$ in the image recorded at time $t$ is equal to the intensity of the point ( $x$ $+u, y+v)$ at time $t+\Delta t$.

As it is assumed that the intensity at a point on the object surface does not change, only the position within the image changes, a constraint on the intensity can be introduced; this is known as the constant brightness constraint. This can be expressed mathematically by differentiation of Eq. (3) using the chain rule, yielding:

$E_{x} u+E_{y} v+E_{t}=0$

where $E_{x}=\delta E / \delta x, E_{y}=\delta E / \delta y$, and $E_{t}=\delta E / \delta t . \Delta t$ is defined as equal to 1 when loading the object statically to simplify the calculation. In the analysis, the error term $\xi_{b}$ is calculated as a measure of the departure from the constant brightness constraints calculated using:

$\xi_{b}=E_{x} u+E_{y} v+E_{t}$

In the analysis of the pair of images, the minimum value of the error term $\xi_{b}$ is determined by a least squares technique.

The OFF technique also requires that the smoothness constraint is met, i.e., that neighboring points on the surface have similar velocities 7 and that higher-order image spatial gradients are similar. ${ }^{19}$ This is also achieved by minimization of an error term using a least squares technique. A directional smoothness constraint was introduced by $\mathrm{Fu}$ and Pridmore, ${ }^{20}$ which considers that the directions of the deforming vectors of the neighboring points on the surface should vary smoothly. This modified smoothness constraint was originally introduced for the investigation of discontinuous surfaces that are found in fluid flows, but is also suitable for the investigation of small local deformations. Mathematically, the error term for the modified smoothness constraint $\xi_{c}$ is found by: 
$\xi_{c}^{2}=\left[\frac{\delta(u / v)}{\delta x}\right]^{2}+\left[\frac{\delta(u / v)}{\delta y}\right]^{2}$

This error term is also minimized in the image analysis. The directional smoothness constraint was also applied to the estimation of the velocity distribution in Schlieren velocimetry. ${ }^{21}$

In the OFF technique, the overall error contribution $\xi$ from the constant brightness constraint error term and the modified smoothness constraint error term across the image is calculated:

$\xi^{2}=\iint\left(\varphi^{2} \xi_{c}^{2}+\xi_{b}^{2}\right) d x d y$,

where $\varphi$ is a scaling factor that is used balance the contribution from the $\xi_{b}$ and $\xi_{c}$ error terms. Using the calculus of variation, $\partial\left(\xi^{2}\right) / \partial u=0$ and $\partial\left(\xi^{2}\right) / \partial v=0$, and the numerical approximations $\nabla^{2} u=\bar{u}-u$ and $\nabla^{2} v=\bar{v}-v$, a pair of equations for each point in the image are obtained:

$$
\begin{aligned}
\left(E_{x}^{2} v^{2}+E_{y}^{2} u^{2}+\varphi^{2}\right)(u-\bar{u})= & -\left(v^{2}+1\right) E_{x} \\
& \times\left(E_{x} \bar{u}+E_{y} v+E_{t}\right),
\end{aligned}
$$

$$
\begin{aligned}
\left(E_{x}^{2} v^{2}+E_{y}^{2} u^{2}+\varphi^{2}\right)(v-\bar{v})= & -\left(u^{2}+1\right) E_{x} \\
& \times\left(E_{x} u+E_{y} \bar{v}+E_{t}\right) .
\end{aligned}
$$

An iterative process based on the Gauss-Seidel method produces the following results. ${ }^{20}$

$u_{n+1}=\bar{u}_{n}-\left(v_{n}^{2}+1\right) E_{x} \frac{\left(E_{x} \bar{u}_{n}+E_{y} v_{n}+E_{t}\right)}{\left(E_{x}^{2} v_{n}^{2}+E_{y}^{2} u_{n}^{2}+\varphi^{2}\right)}$,

$v_{n+1}=\bar{v}_{n}-\left(u_{n}^{2}+1\right) E_{y} \frac{\left(E_{x} u_{n}+E_{y} \bar{v}_{n}+E_{t}\right)}{\left(E_{x}^{2} v_{n}^{2}+E_{y}^{2} u_{n}^{2}+\varphi^{2}\right)}$,

where the local weighted averages are given by:

$$
\begin{aligned}
& \bar{u}=-\frac{1}{4}+\sum_{\alpha=-1}^{\alpha=1} \sum_{\beta=-1}^{\beta=1}\left[\frac{u(x+\alpha, y+\beta)}{(4+2|\alpha| \sqrt{2}+2|\beta| \sqrt{2})}\right], \\
& \bar{v}=-\frac{1}{4}+\sum_{\alpha=-1}^{\alpha=1} \sum_{\beta=-1}^{\beta=1}\left[\frac{v(x+\alpha, y+\beta)}{(4+2|\alpha| \sqrt{2}+2|\beta| \sqrt{2})}\right],
\end{aligned}
$$

and the derivatives $E_{x}, E_{y}$, and $E_{t}$ are calculated by:

$$
\begin{aligned}
& E_{x}=\frac{(2 \alpha-1)}{4} \sum_{\alpha=0}^{\alpha=1} \sum_{\beta=0}^{\beta=1} \sum_{\chi=0}^{\chi=1} E(x+\alpha, y+\beta, t+\chi), \\
& E_{y}=\frac{(2 \beta-1)}{4} \sum_{\alpha=0}^{\alpha=1} \sum_{\beta=0}^{\beta=1} \sum_{\chi=0}^{\chi=1} E(x+\alpha, y+\beta, t+\chi), \\
& E_{t}=\frac{(2 \chi-1)}{4} \sum_{\alpha=0}^{\alpha=1} \sum_{\beta=0}^{\beta=1} \sum_{\chi=0}^{\chi=1} E(x+\alpha, y+\beta, t+\chi) .
\end{aligned}
$$

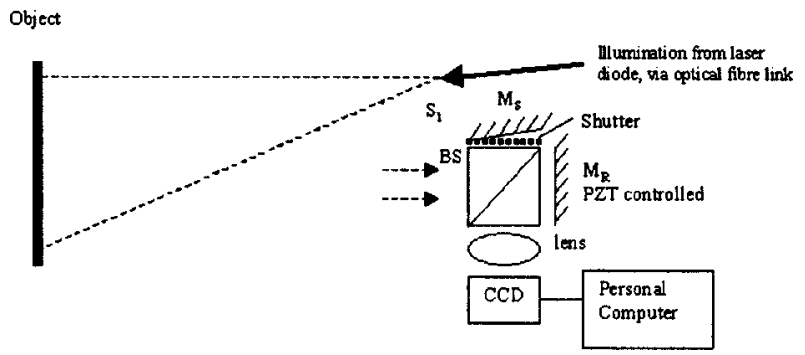

Fig. 1 The experimental layout of the combined shearography and digital speckle photography instrument. The optical source $S_{1}$ is located as close as possible to the normal of the object surface without obstructing the field of view of the camera. BS is the beamsplitter; $M_{S}$ is the shearing mirror, which is adjustable for angle and direction of tilt; and $M_{R}$ is the reference mirror with PZT control to perform the phase stepping.

The displacement field calculated by this OFF technique is differentiated numerically in the $x$ and $y$ directions to obtain the four in-plane displacement gradient components $\delta u / \delta x, \delta u / \delta y, \delta v / \delta u$, and $\delta v / \delta y$.

\section{Combined Shearography and Digital Speckle Photography}

The strain tensor $S$ is composed of nine components: six surface strain displacement gradient components and three bulk strain components ${ }^{22}$ :

$$
S=\left[\begin{array}{ccc}
\frac{\delta u}{\delta x} & \frac{1}{2}\left(\frac{\delta u}{\delta y}+\frac{\delta v}{\delta x}\right) & \frac{1}{2}\left(\frac{\delta u}{\delta z}+\frac{\delta w}{\delta x}\right) \\
\frac{1}{2}\left(\frac{\delta u}{\delta y}+\frac{\delta v}{\delta x}\right) & \frac{\delta v}{\delta y} & \frac{1}{2}\left(\frac{\delta v}{\delta z}+\frac{\delta w}{\delta y}\right) \\
\frac{1}{2}\left(\frac{\delta u}{\delta z}+\frac{\delta w}{\delta x}\right) & \frac{1}{2}\left(\frac{\delta v}{\delta z}+\frac{\delta w}{\delta y}\right) & \frac{\delta w}{\delta z}
\end{array}\right] .
$$

Single-axis shearography can measure the out-of-plane displacement gradient components $\delta w / \delta x$ and $\delta w / \delta y$. Singleaxis digital speckle photography can measure the in-plane displacement gradient components $\delta u / \delta x, \delta u / \delta y, \delta v / \delta x$, and $\delta v / \delta y$. The surface strain can therefore be fully characterized using independently measured components from the shearography and digital speckle photography techniques.

\section{Experimental Details}

A single-axis combined shearography and digital speckle photography instrument was constructed. The main components were laser illumination, a shearography interferometer head with a shutter to block the shearing mirror and the facility to switch between the $d x$ and $d y$ shear directions, and image processing software to perform the digital speckle photography analysis. The experimental layout is shown in Fig. 1.

A single-mode laser diode (100 mW, $800 \mathrm{~nm})$, with optical fiber beam delivery to the source position, was used to illuminate the surface of the object under investigation. Optical fiber beam delivery provided spatial filtering of the beam and allowed flexible positioning of the source. A lens 
located at the distal end of the optical fiber was used to expand the beam. The beam expansion lens was located $600 \mathrm{~mm}$ from the object and $90 \mathrm{~mm}$ below the normal to the center of the object surface.

The shearography head contained a shearing Michelson interferometer, a camera lens, and a charge-coupled device (CCD) camera (CA-D4-0512A, 75-Hz frame rate, 512 $\times 512$ pixels, 8 bit, Dalsa, Waterloo, ON). The shearing interferometer was composed of a beamsplitter, a shearing mirror, and a reference mirror mounted on a piezoelectric transducer to perform the phase stepping. The shutter was used to block the shearing mirror to allow speckle patterns to be recorded for the DSP analysis. The shearing mirror was adjustable to select different magnitudes and directions of applied shear. A PC running LabVIEW software (National Instruments Corporation, Austin, TX) controlled image capture by the CCD camera and phase stepping.

To perform a measurement, reference images were recorded before object deformation and deformed images after object deformation. Initially, the shear was applied in the horizontal $x$ direction. The object was illuminated by the laser and a reference DSP image was recorded with the shearing mirror blocked to prevent the formation of interferometric speckles. After unblocking the shearing mirror, three reference shearography phase-stepped speckle interferograms were recorded, with phase steps of $-2 \pi / 3,0$, and $2 \pi / 3$ radians. The object was then deformed and a single deformed speckle interferogram was recorded at a phase step of 0 radians. The shearing mirror was again blocked and a deformed DSP was recorded. To record images from the vertical $y$ shearing direction, the tilt of the shearing mirror was changed and the reference and deformed interferogram image capture process was repeated.

Wrapped phase maps were calculated from the shearography interferograms as described in Eq. (2) using the LabVIEW program. The wrapped phase maps were unwrapped using ISTRA software ${ }^{23}$ (Dantec-Ettemeyer $\mathrm{GmbH}$, Ulm, Germany). The unwrapped maps were converted to a measurement of strain using Eq. (1). The digital speckle photography images were processed using the OFF technique described in Sec. 3.

\section{Results}

A flat plate aluminum test object with dimensions $200 \times 200$ $\mathrm{mm}$, clamped around the perimeter and painted white (vinyl matt Dulux, Slough, Berkshire, UK), was deformed by a micrometer screw gauge located behind the object, and the deformation of the object was out-of-plane by $10 \mu \mathrm{m}$ approximately in the center of the field of view. The applied shear had a magnitude of $2.5 \mathrm{~mm}$ in both the horizontal and vertical directions. Interferometric speckle patterns recorded before and after object deformation were used for the shearography analysis. Noninterferometric speckle patterns recorded before and after object deformation were used for the OFF analysis.

Figure 2 shows wrapped phase maps generated using the shearography technique described in Sec. 2. Figure 3 shows the noninterferometric speckle patterns recorded before and after object deformation and the displacement field calculated using the OFF, described in Sec. 3.

The unwrapped phase maps are scaled using the optical wavelength, the shear magnitude, and the angles of illumi-

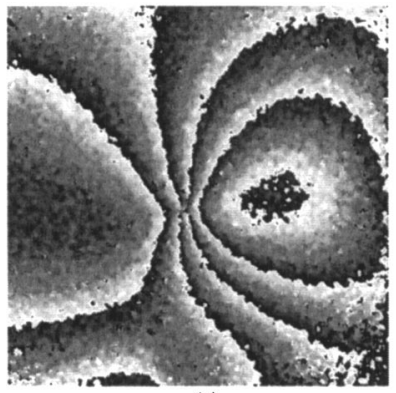

(a)

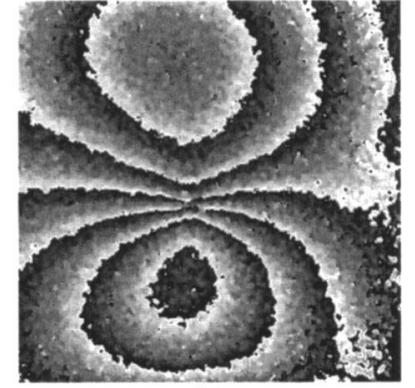

(b)
Fig. 2 The wrapped phase maps, determined using the shearography technique, for (a) a horizontal shear of $2.5 \mathrm{~mm}$ and (b) a vertical shear of $2.5 \mathrm{~mm}$.

nation and viewing using Eq. (1) to yield the out-of-plane displacement gradient components $\delta w / \delta x$ and $\delta w / \delta y$. The displacement field, shown in Fig. 3, is differentiated numerically and scaled to yield the in-plane displacement gradient components $\delta u / \delta x, \delta v / \delta x, \delta u / \delta v$, and $\delta v / \delta y$. The calibration process for the OFF technique was presented previously. ${ }^{24}$ An aluminum specimen was loaded using a tensile test machine, and calibration measurements of the surface strain were made using resistance strain gauges. The specimen was loaded to give displacement gradients

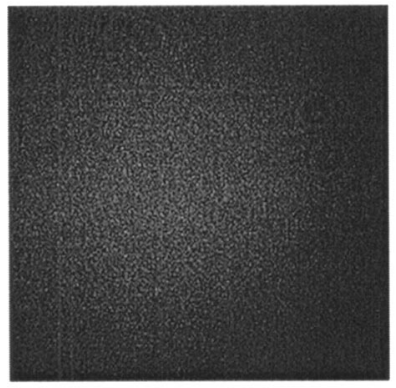

(a)

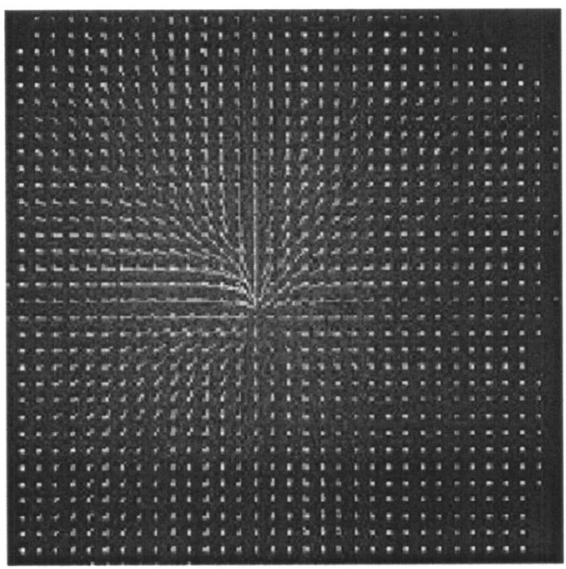

(c)

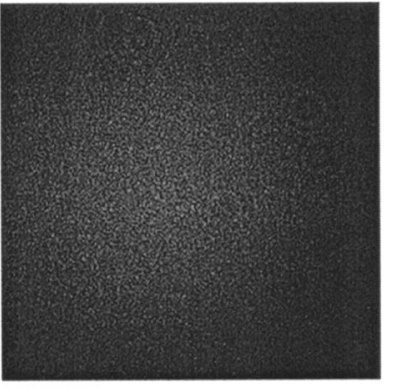

(b) Fig. 3 Noninterferometric speckle patterns recorded (a) before and
(b) after application of an out-of-plane displacement of $10 \mu \mathrm{m}$ approximately in the center of the field of view. (c) Shows the displacement field calculated from images (a) and (b) using the OFF technique. 


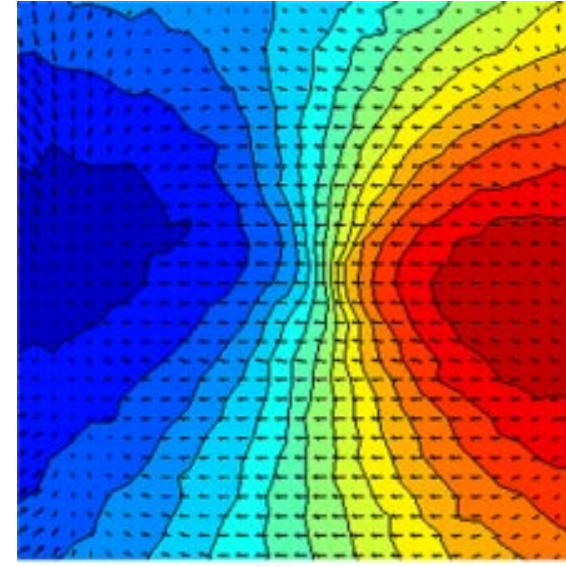

(a)

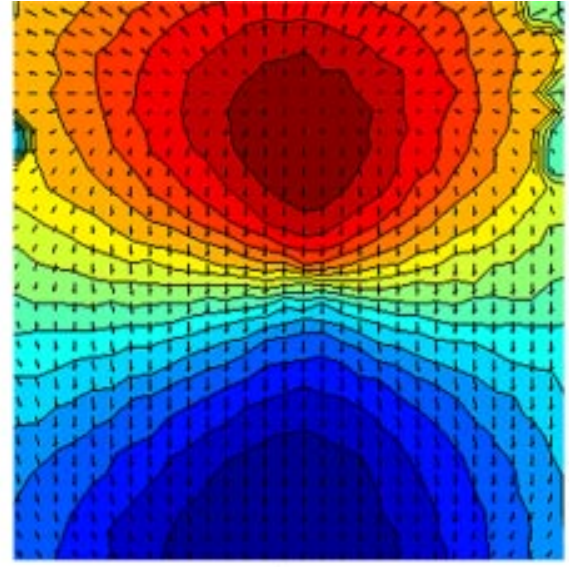

(b)

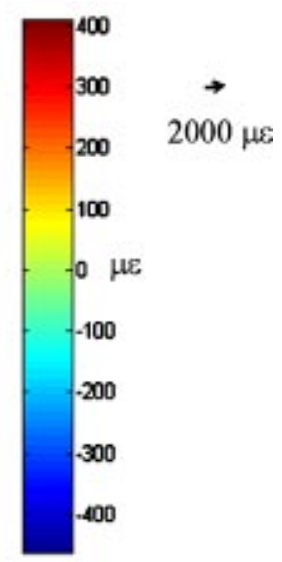

(c)

Fig. 4 The surface strain field for (a) the $\delta / \delta x$ displacement gradient components and (b) the $\delta / \delta y$ displacement gradient components. The out-of-plane displacement gradient components are represented by the color scale (grayscale) in (c). The in-plane displacement gradient components are represented by arrows, where (c) shows the relationship between the length of the arrow and the displacement gradient. The direction of the arrow shows the results of the $\delta u / \delta x$ and $\delta v / \delta x$ components and the results of the $\delta u / \delta y$ and $\delta v / \delta y$ components.

between 0 and 10000 microstrains. The rms error determined was $\pm 500 \mu \mathrm{m}$.

Figure 4 shows the surface strain maps generated by combining the data from the digital speckle photography and the shearography techniques. In Fig. 4(a) the $\delta u / \delta x$ and $\delta v / \delta x$ in-plane displacement gradient components are represented by vectors, and the $\delta w / \delta x$ out-of-plane displacement gradient component is represented by a color map. In Fig. 4(b) the $\delta u / \delta y$ and $\delta v / \delta y$ in-plane displacement gradient components are represented by vectors, and the $\delta w / \delta y$ out-of-plane displacement gradient component is represented by a color map. The in-plane displacement gradient vectors represent magnitudes of microstrains between 500 and $2000 \mu \epsilon$. The out-of-plane displacement gradients are between -400 and $+400 \mu \epsilon$.

\section{Discussion}

The combined shearography and speckle pattern instrument has been used successfully to fully characterize the surface strain. For this experimental layout, the shearography technique, with phase measurements made to $1 / 10$ fringe, has an accuracy of $\pm 20 \mu \epsilon$. The shearography technique has an order of magnitude greater sensitivity than the OFF technique. The sensitivity of both techniques is suitable for the investigation of the mechanical properties of materials and components.

The combined shearography and DSP technique is most useful where the optical access is limited to a single illumination and viewing direction. This is particularly important when investigating surface strains within machinery and structures where optical access is compromised. The technique as presented is suitable for surface strain measurements on flat surfaces normal to the instrument illumination and viewing directions, as this is where the in-plane and out-of-plane direction vectors of the instrument coincide with the in-plane and out-of-plane direction vectors of the object. When illumination and viewing at the normal to the object surface is not possible, a coordinate transformation would be required to obtain the orthogonal components.

One of the features of this instrument is the ability to perform independent measurements of the in-plane and outof-plane displacement gradient components. This minimizes the propagation of errors in the calculations. For example, when performing a multicomponent measurement using multicomponent shearography alone, errors in one of the measured channels can propagate to all the calculated components in the coordinate transformation process. ${ }^{12}$

\section{Conclusions}

In this work, a single-axis multicomponent surface strain measurement instrument is demonstrated. The technique combines the speckle interferometry technique of shearography and the digital speckle photography technique of optical flow field. The full-surface strain measurement from a single illumination and viewing direction is demonstrated.

\section{Acknowledgments}

This work is supported by the Engineering and Physical Sciences Research Council, United Kingdom (GR/N11872/ 01), Augusta-Westland, the Civil Aviation Authority, United Kingdom, and by the Royal Society, United Kingdom.

\section{References}

1. J. A. Leendertz and J. N. Butters, "An image-shearing speckle pattern interferometer for measuring bending moments," J. Phys. E 6, 11071110 (1973).

2. Y. Y. Hung, "Shearography: a new optical method for strain measurement and nondestructive testing," Opt. Eng. 21(2), 391-395 (1982).

3. M. A. Sutton, W. J. Wolters, W. H. Peters, W. F. Ranson, and S. R. McNeill, "Determination of displacements using an improved digital correlation method," Image Vision Computing 1, 133-139 (1983).

4. D. J. Chen and F. P. Chiang, "Optimal sampling and range of measurement in displacement-only laser-speckle correlation," Exp. Mech. 32, 145-153 (1992).

5. S. Noh and I. Yamaguchi, "Two-dimensional measurement of strain 
distribution by speckle correlation," Jpn. J. Appl. Opt. 31, L1299L1301 (1992).

6. M. Sjödahl and L. R. Benckert, "Electronic speckle photography: analysis of an algorithm giving the displacement with subpixel accuracy," Appl. Opt. 32, 2278-2284 (1994).

7. B. K. P. Horn and B. G. Schunck, "Determining optical flow," J. Artif. Intell. Res. 17, 185-203 (1981).

8. A. Andersson, N. Krishna Mohan, M. Sjödahl, and N.-E. Molin, "TV shearography: quantitative measurement of shear magnitude fields by use of digital speckle photography," Appl. Opt. 39, 2565-2568 (2000).

9. N. E. Molin, M. Sjödahl, P. Gren, and A. Svanbro, "Speckle photography combined with speckle interferometry," Opt. Lasers Eng. 41, $673-686$ (2004)

10. L. X. Yang, W. Steinchen, M. Schuth, and G. Kupfer, "Precision measurement and nondestructive testing by means of digital phase shifting speckle pattern and speckle pattern shearing interferometry," Measurement 16, 149-160 (1995)

11. H. A. Aebischer and S. Waldner, "Strain distributions made visible with image-shearing speckle pattern interferometry," Opt. Lasers Eng. 26, 407-420 (1997)

12. S. W. James and R. P. Tatam, "Time-division-multiplexed 3D shearography," Proc. SPIE 3744, 394-403 (1999).

13. L. Larsson, M. Sjödahl, and F. Thuvander, "Microscopic 3-D displacement field measurements using digital speckle photography," Opt. Laser. Eng. 41, 767-777 (2004).

14. Y. Y. Hung and C. Y. Liang, "Image-shearing camera for direct measurement of surface strain," Appl. Opt. 18, 1046-1051 (1979).

15. K. Creath, "Temporal phase measurement methods," in Interferogram Analysis, Digital Fringe Measurement Methods, D. W. Robinson and G. T. Reid, Eds., pp. 94-140, Institute of Physics, Bristol (1993).

16. G. Vendroux and W. G. Knauss, "Submicron deformation field measurement: Part 2. Improved digital image correlation," Exp. Mech. 38, 86-92 (1998).

17. D. W. Manthey and D. Lee, "Vision-based surface strain measurement system," JOM 47, 46-49 (1995).

18. T. C. Chu, W. F. Ranson, M. A. Sutton, and W. H. Peters, "Applications of digital-image-correlation techniques to experimental mechanics," Exp. Mech. 25, 232-244 (1985).

19. S. Uras, F. Girosi, A. Verri, and V. Torre, "A computational approach to image perception," Biol. Cybern. 60, 79-87 (1988).

20. S. Fu and T. P. Pridmore, "Image flow field detection," Third Intl. Conf. Sig. Process. (ICSP'96) 2, 1090-1093 (1996).

21. S. Fu and Y. Wu, "Detection of velocity distribution of a fluid flow using sequences of Schlieren images," Opt. Eng. 40(8), 1661-1666 (2001).

22. H. Cabannes, General Mechanics, trans. S. P. Sutera, Blaisdell Publishing Company, Waltham, MA (1968).

23. A. Ettemeyer, U. Neuport, H. Rottenkolber, and C. Winter, "Schnelle und robuste bildanalyse von streifenmustern-ein wichtiger schritt der automation von holografischen prozessen," 1st Intl. Workshop Auto. Process. Fringe Patt., pp. 23-31 (1989).
24. S. Fu, D. Furfari, and P. E. Irving, "Pseudo speckle pattern photography-algorithm development and experimental calibration,' Proc. SPIE 5058, 266-272 (2002).

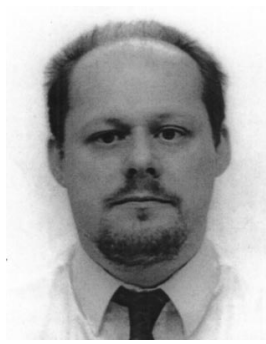

Roger M. Groves is a research fellow in the Centre for Photonics and Optical Engineering at Cranfield University. He has a BSc in chemistry from the University of Wales, Aberystwyth, an MSc in applied physics with distinction from the University of Hertfordshire, United Kingdom, and a $\mathrm{PhD}$ in optical instrumentation from Cranfield University. He has six years of experience in development of speckle metrology instrumentation for the measurement of surface strain, shape, and for vibration analysis. He has 20 journal and conference publications.

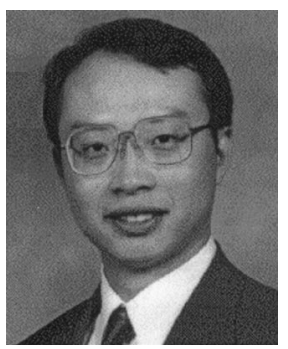

Shan Fu is a lecturer in the Centre for Computer Vision and Image Processing at Cranfield University, United Kingdom. He received a BEng degree in electronic engineering from Northwestern Polytechnic University in China, and a PhD degree in Heriot-Watt University, United Kingdom. All his research relates to computer vision, image processing, and related system development.

Stephen W. James Biography and photograph not available.

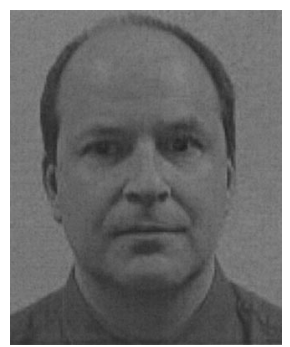

Ralph P. Tatam is a professor of engineering photonics in the School of Engineering at Cranfield University and heads the optical sensors group. He has been active in optical and optical-fiber-based instrumentation and sensing for nearly 20 years. His current research interests include optical flow measurement, speckle interferometry, and optical fiber sensors. He graduated with a BSc in physics and chemistry from Exeter University, United Kingdom, in 1981, and received a PhD in physics (CNAA) in 1986. Following four years at the physics laboratory at the University of Kent, he moved to Cranfield University. He has published more than 250 papers and has been involved with numerous national and international conferences. 